\title{
Creatividad y adolescencia: Diferencias según género, curso y nivel cognitivo
}

\author{
Víctor Manuel Belmonte-Lillo y Ana Isabel Parodi \\ Universidad de Alicante (España)
}

\begin{abstract}
El estudio analiza si existen diferencias en los niveles de creatividad de los adolescentes en función del género, del nivel cognitivo y del curso académico respectivamente. La muestra se compone de 670 adolescentes (edades entre 12 y 16 años). El análisis factorial de la variable creatividad de los datos reportó dos factores diferenciados: pensamiento divergente (aglutina los componentes creativos de fluidez, flexibilidad y originalidad) y elaboración. En la variable aptitud intelectual se establecieron tres grupos de inteligencia (baja, media y alta). En relación a los resultados, no se encontraron diferencias estadísticamente significativas entre géneros para el pensamiento divergente pero sí en el factor elaboración a favor de las chicas lo que indicaría nivel sensiblemente mayor de desarrollo, complejidad y detalle de una producción o idea. No se hallaron diferencias en función del nivel intelectual para el factor de pensamiento divergente y sí en el factor de elaboración a favor de los grupos de mayor capacidad intelectiva, lo que apoyaría en parte la independencia de los constructos de creatividad e inteligencia. Por último, la variable de curso nos muestra un decrecimiento del pensamiento divergente y la elaboración conforme se avanza en la ESO, salvo que este último factor presentará un cénit en el último curso de la enseñanza obligatoria.
\end{abstract}

Palabras clave: Creatividad, adolescencia, diferencias de género, nivel cognitivo.

Creativity and adolescence: Differences according to gender, course and cognitive level. The study analyzes whether there are differences in the levels of creativity of adolescents according to gender, cognitive level and academic course respectively. The sample consists of 670 adolescents (ages 12 to 16 ). The factorial analysis of the variable creativity reported two differentiated factors: divergent thinking (fluency, flexibility and originality components) and elaboration. In the intellectual aptitude variable, three intelligence groups were established (low, medium and high). In relation to the results, no statistically significant differences were found between genders for divergent thinking but in the elaboration factor in favor of the girls, which would indicate a significantly higher level of development, complexity and detail of a production or idea. No differences were found depending on the intellectual level for the divergent thinking factor. In the elaboration factor were found differences in favor of groups with greater intellectual capacity, which would partly support the independence of the constructs of creativity and intelligence. Lastly, the course variable shows a decrease in divergent thinking and the elaboration of progress in ESO, except that this last factor will present a zenith in the last year of ESO.

Keywords: Creativity, adolescence, gender differences, cognitive level.

Correspondencia: Víctor Manuel Belmonte-Lillo. Universidad de Alicante. Apartado de correos 99. C.P.: 03080. Alicante (España). E-mail: victor.belmonte@ua.es 
La creatividad es una cualidad que diferencia al ser humano del resto de seres vivos, ya que no sólo somos capaces de analizar y entender la realidad sino de imaginar nuevas formas de la misma. Pese a su importancia, no será hasta mitad del siglo XX cuando reciba la atención merecida por parte de la ciencia psicológica que ha relacionado el constructo con la resolución de problemas, la inteligencia, la personalidad y/o un funcionamiento particular de la actividad cerebral. Considerada actualmente como una cualidad humana fundamental y con gran valor adaptativo para nuestra especie, numerosas teorías han abordado su definición. Destacamos la perspectiva psicométrica desarrollada por Joy Paul Guilford y por su discípulo Paul Torrance. Guilford (1967) enmarca la creatividad dentro de la inteligencia y otorga importancia a un tipo de pensamiento relacionado con la creatividad, el pensamiento divergente; además sus trabajos permitieron delimitar los factores implicados en la conducta creativa (sensibilidad a los problemas, fluidez, flexibilidad, originalidad, elaboración y capacidad de redefinición). Por su parte, Torrance (1974) realizará grandes aportes en la medición de la creatividad con su elaboración del Torrance Test of Creative Thinking (TCTT), operativizando el constructo de manera que nos permite estudiarlo a través de cuatro factores: fluidez, flexibilidad, originalidad y elaboración; y abogando también por una creatividad como facultad educable.

Numerosas investigaciones se han centrado en el estudio de la relación entre inteligencia y creatividad, en este sentido, destacamos la Teoría del Umbral propuesta por Torrance (1962) en la que los constructos de inteligencia y creatividad se relacionarían o no en función del nivel cognitivo. Algunas investigaciones no apoyan dicha teoría (Kim, 2005; Preckel, Holling, y Wiese, 2006) o no hallan relación general entre creatividad e inteligencia (Silvia, 2008; Naderi et al., 2010; López-Martínez y Navarro-Lozano, 2010; Miranda, Almedia, Morais, y Guisade, 2012). Por su parte, otros autores sí apuntan a la relación entre creatividad e inteligencia (Nusbaum y Silvia, 2010; Silvia y Rogers, 2012). Respecto a diferencia de géneros en la creatividad, Baer y Kaufman (2008) realizan una revisión de la literatura científica concluyendo que no existen diferencias ligadas a esta variable. Por su parte, el desarrollo de la creatividad a través de los diferentes cursos escolares también ha sido estudiado ofreciendo diversos resultados (Smith y Carlsson, 1990; Runco y Sakamoto, 1999; Claxton, Pannells, y Rhoads, 2005; Kim, 2011).

En la misma línea de estudio que las investigaciones expuestas y desde un enfoque psicométrico, el presente trabajo tiene como objeto delimitar si existe diferencia en la creatividad dentro de la etapa de la adolescencia considerando el género, el nivel cognitivo y el curso académico de los participantes. 


\section{MÉTODO}

\section{Participantes}

La muestra de estudio está compuesta por 670 alumnos de Educación Secundaria Obligatoria (ESO) pertenecientes a 3 centros privados-concertados de la provincia de Alicante: 106 alumnos de un colegio de la localidad de San Juan, 293 alumnos de un colegio de la localidad de Alicante, 271 de un colegio de la localidad de Elda. De ellos un $46.6 \%$ son varones. Los alumnos tienen edades comprendidas entre los 12 y 16 años $(M=13.86 ; D T=1.23)$. De total de la muestra un $31.9 \%$ pertenecen a $1^{\circ}$ ESO, un $26.9 \%$ a $2^{\circ}$ de ESO, un $23.6 \%$ a $3^{\circ}$ de ESO y un $17.6 \%$ a $4^{\circ}$ de ESO. La selección de la muestra se realizó mediante un muestreo de carácter incidental.

\section{Instrumentos}

En el estudio se utilizaron diferentes instrumentos atendiendo a los constructos de creatividad y nivel cognitivo:

Para valorar la creatividad se utilizó el subtest 3 del Test de Pensamiento Creativo de Torrance (TTCT, Subtest 3 versión Figurativa; Torrance, 1974). Éste se compone de 30 pares de líneas paralelas y pretende evaluar las producciones creativas a través de dibujos y composiciones. La tarea consiste en hacer tantos dibujos como sea posible usando esas líneas. Las dimensiones medidas son: Fluidez, Flexibilidad, Originalidad y Elaboración.

Una vez obtenida la puntuación global, se procedió a establecer categorías en función del nivel de creatividad evaluado. Se obtuvieron tres: el grupo alta creatividad supone un $25.6 \%$ de la muestra y se estableció desde el valor 22.83 obtenido en la medición del TTCT; el grupo de creatividad media supone un $49.2 \%$ de la muestra, cuyos resultados están entre 11.33 y el 22.83 ; por último, el grupo de baja creatividad supone un $25.3 \%$ de la muestra, sus valores son inferiores a 11.33.

Por otra parte, para medir el factor de inteligencia psicométrica se utilizaron dos pruebas. El Test de Aptitudes Diferenciales (DAT-5; Bennett et al., 2000) es un test de rendimiento diseñado para evaluar un conjunto de habilidades mentales: razonamiento verbal (habilidad para descubrir relaciones entre palabras), razonamiento numérico (habilidad para enfrentarse a las tareas de razonamiento matemático), razonamiento abstracto (medida no verbal de la habilidad para razonar), razonamiento mecánico (mide la capacidad de comprensión de principios básicos mecánicos de maquinaria, herramientas y movimientos), relaciones espaciales (habilidad para visualizar un objeto de tres dimensiones a partir de un modelo bidimensional e imaginar cómo aparecería este objeto si sufriera una rotación espacial), ortografía (grado en que los estudiantes pueden escribir y deletrear correctamente palabras de uso común), rapidez y exactitud perceptiva (habilidad para comparar y comprobar de forma rápida y 
precisa documentos escritos). Se aplicó también la Batería de Aptitudes Diferenciales y Generales: prueba complementaria Memoria de relato oral (BADyG-M; Yuste, Martínez y Galve, 1998); se ha optado por aplicar dicha prueba, con tal de obtener una medición de la memoria de los sujetos. Esta medición nos permite completar la valoración del factor de inteligencia general realizado con el DAT-5 (subpruebas de razonamiento). Para obtener un índice de inteligencia general, se procedió a realizar una suma de las puntuaciones directas obtenidas por cada sujeto en las diferentes subpruebas de razonamiento (DAT-5) y la prueba de memoria (BADyG-M). No se consideraron las subpruebas de Ortografía y Rapidez y Exactitud Perceptiva (DAT-5).

Además de obtener el índice general de inteligencia de cada uno de los sujetos de la muestra, se realizó también una agrupación en función del nivel cognitivo mediante el procedimiento de cuartiles aplicado a la distribución de la población en función de sus puntuaciones. Siguiendo la metodología de formación de grupos contrastantes (Almeida y Freire, 2008) se describieron tres grupos diferenciados: grupo de baja inteligencia, Q1 (27\% de la muestra) que obtiene unos resultados inferiores a PD 106; el grupo de inteligencia alta, situado por encima del Q3 (27\% de la muestra) cuyas puntuaciones se sitúan por encima de PD 149; y el grupo inteligencia media, entre el Q1 y Q3 (46\% de la muestra).

\section{Procedimiento}

Por un lado, se realizó una revisión y análisis bibliográfico sobre los tópicos de estudio y sus instrumentos de medición.

Por otro, se estableció una planificación para la evaluación mediante los instrumentos descritos de los constructos creatividad e inteligencia. En un primer momento se solicitó a las diferentes direcciones de los Centros la participación en el estudio. Dada su conformidad, se informó a las familias mediante una circular sobre el estudio que se iba a realizar. Todas se mostraron colaboradoras. Conjuntamente con la Jefatura de Estudios de los diferentes centros, se estableció un calendario y horario de aplicación de pruebas. En los días acordados, en diferentes sesiones del horario lectivo, se procedió a la aplicación de las diferentes pruebas psicométricas, llevándose a cabo de forma grupal y dentro del aula de referencia. En todo momento, se siguieron las instrucciones marcadas por los autores para los diferentes instrumentos.

Una vez realizada la recogida de datos, se procedió a la corrección, codificación, registro y preparación de datos de los alumnos en soporte informático para su depuración y análisis posterior mediante el paquete estadístico SPSS versión v.20.0 para Mac Os. Por último, se estableció el diseño para el análisis de los datos, teniendo en cuenta los procedimientos estadísticos convenientes en función de los objetivos propuestos. Se analizaron los datos, mediante el programa estadístico citado, 
acometiendo cada uno de los objetivos planteados. Procediendo, a partir de los datos obtenidos, a la elaboración del apartado de discusión y conclusiones.

\section{Análisis de datos}

El análisis de datos abordó distintos procedimientos en función de los objetivos e incluyeron:

Análisis factorial exploratorio utilizando el método de extracción de componentes principales con rotación Varimax para la determinación de la estructura interna del TCTT.

Análisis de diferencia de medias mediante la prueba $t$ de Student y el análisis de varianza con el objetivo de comprobar la existencia de diferencias estadísticamente significativas en creatividad según género, nivel intelectual y curso de los participantes.

\section{RESULTADOS}

\section{Análisis factorial del TCTT}

La tabla 1 muestra los resultados del análisis de componentes principales con rotación varimax realizados para todas las variables de creatividad valoradas en el tercer subtest del TCTT (fluidez, flexibilidad, originalidad y elaboración).

La medida de adecuación muestral KMO fue de .763, y la prueba de esfericidad de Bartlett resultó significativa $\left(\chi^{2}=2722,241\right.$; gl $\left.=6, \mathrm{p}<.001\right)$. Siguiendo la información aportada por el gráfico de sedimentación (gráfica 1) se solicitó la extracción de dos componentes que explicaron en su conjunto un $95.86 \%$ de la varianza. El primer componente agrupó las dimensiones de fluidez, flexibilidad y originalidad y explicó un $69 \%$ de la varianza, y podría ser denominado como pensamiento divergente. La dimensión de elaboración constituyó el segundo componente explicando un $26.86 \%$ de la varianza.

Gráfica 1. Gráfico de sedimentación TCTT (subtest 3)

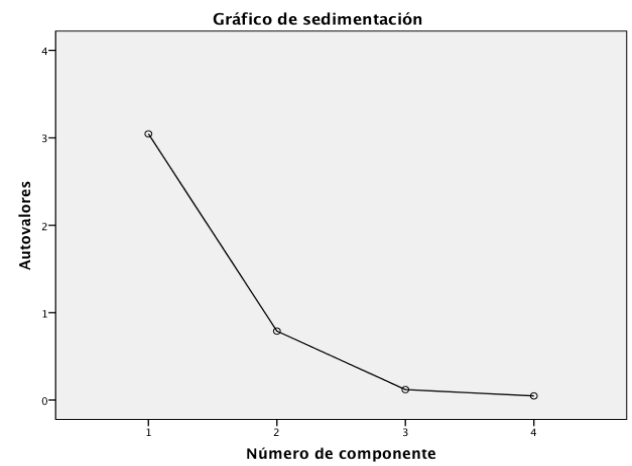


Tabla 1. Estructura factorial TCTT (subtest 3)

\begin{tabular}{lccc}
\hline & \multicolumn{2}{c}{ Componente } & \multirow{2}{*}{ H2 } \\
\cline { 2 - 3 } & 1 & 2 & .969 \\
\hline TTCT Fluidez & .969 & & .938 \\
\hline TTCT Flexibilidad & .944 & .928 \\
\hline TTCT Originalidad & .943 & .980 & 1 \\
\hline TTCT Elaboración & & .789 & - \\
\hline Autovalores & 3.046 & $26.86 \%$ & - \\
\hline$\%$ varianza & $69 \%$ & & \\
\hline
\end{tabular}

\section{Análisis inferenciales}

La tabla 2 muestra las medias y desviaciones típicas en creatividad, según el género de los participantes. En general, los chicos obtuvieron medias más elevadas en las variables de pensamiento divergente y las chicas puntuaron más alto en el factor de elaboración.

Tabla 2. Estadísticos descriptivos de las variables de estudio según género

\begin{tabular}{ccccc}
\hline & Chicos $M(D T)$ & $N$ & Chicas $M(D T)$ & $\mathrm{N}$ \\
\hline Pensamiento divergente & $17.27(8.62)$ & 292 & $16.55(8.27)$ & 341 \\
\hline Elaboración & $13.74(8.45)$ & 292 & $15.04(7.87)$ & 341 \\
\hline
\end{tabular}

Para determinar si las diferencias halladas entre las medias de chicos y chicas son lo suficientemente grandes como para poder rechazar la hipótesis nula, se aplicó la prueba paramétrica $t$ de Student para muestras independientes. Los resultados evidenciaron diferencias estadísticamente significativas en elaboración a favor de las chicas (ver tabla 3 ).

\begin{tabular}{lccccc}
\multicolumn{6}{c}{ Tabla 3. Estadísticos descriptivos de las variables de estudio según género } \\
\hline & $\begin{array}{l}\text { Prueba de Levene para } \\
\text { igualdad de varianzas }\end{array}$ & Prueba t para la igualdad de media \\
\cline { 2 - 7 } & $\mathrm{F}$ & Sig. & $\mathrm{t}$ & $\mathrm{gl}$ & Sig. (bilateral) \\
\hline Pensamiento divergente & 1.059 & .304 & 1.067 & 631 & .286 \\
\hline Elaboración & .081 & .77 & -2.012 & 631 & $\mathbf{. 0 4 5}$ \\
\hline
\end{tabular}

La tabla 4 muestra las medias y desviaciones típicas en creatividad según el nivel intelectual de los participantes. Como se aprecia, los alumnos con una inteligencia alta obtuvieron medias más elevadas que los alumnos con media y baja inteligencia en el factor de elaboración. Los alumnos con inteligencia media obtuvieron medias más elevadas a las obtenidas por los alumnos con baja y alta inteligencia en pensamiento divergente. 
BELMONTE-LILLO y PARODI. Creatividad y adolescencia

Tabla 4. Estadísticos descriptivos de las variables de creatividad según grupos de inteligencia

\begin{tabular}{lcccccc}
\hline & \multicolumn{2}{c}{ Grupo 1- CI Bajo } & \multicolumn{2}{c}{ Grupo 2- CI Medio } & \multicolumn{2}{c}{ Grupo 3- CI Alto } \\
\cline { 2 - 7 } & $N$ & Media $(D T)$ & $N$ & Media $(D T)$ & $N$ & Media $(D T)$ \\
\hline Pensamiento diverg. & 161 & $15.81(8.47)$ & 265 & $17.45(8.41)$ & 173 & $16.48(8.07)$ \\
Elaboración & 161 & $12.50(7.35)$ & 265 & $14.79(7.91)$ & 173 & $15.61(8.86)$ \\
\hline
\end{tabular}

A continuación se llevó a cabo un análisis de varianza para estudiar la existencia de diferencias estadísticamente significativas en las variables de la creatividad, según el nivel intelectual de los participantes. Los resultados mostraron diferencias estadísticamente significativas a favor del grupo de inteligencia alta en la variable elaboración (ver tabla 5 y gráfica 2).

Tabla 5. Diferencias entre las variables de creatividad según nivel de inteligencia

\begin{tabular}{lcccc}
\hline \multicolumn{1}{c}{ Variable dependiente } & Gl & F & Sig. & Diferencias entre grupos \\
\hline Pensamiento divergente & 2.596 & 2.045 & .130 & \\
\hline Elaboración & 2.596 & 6.751 & $\mathbf{. 0 0 1}$ & $1<2,1<3$ \\
\hline
\end{tabular}

Gráfica 2. Gráfica de diferencias significativas en elaboración según nivel de inteligencia

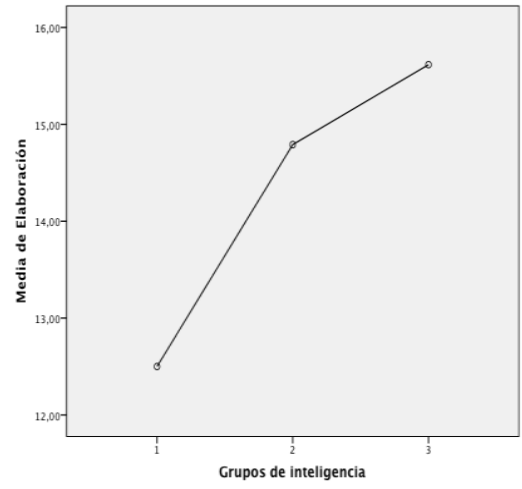

Se presentan (ver tabla 6) las medias y desviaciones típicas en las variables de creatividad según el curso al que pertenecen los participantes.

Tabla 6. Estadísticos descriptivos de las variables de estudio según curso académico

\begin{tabular}{lcccccccc}
\hline & \multicolumn{2}{c}{$1^{\circ}$ ESO } & \multicolumn{2}{c}{$2^{\circ}$ ESO } & \multicolumn{2}{c}{$3^{\circ}$ ESO } & \multicolumn{2}{c}{$4^{\circ}$ ESO } \\
\cline { 2 - 9 } & $N$ & Media $(D T)$ & $N$ & Media $(D T)$ & $N$ & Media $(D T)$ & $N$ & Media $(D T)$ \\
\hline Pensamiento divergente & 204 & $16.96(8.17)$ & 168 & $18.27(8.16)$ & 146 & $17.00(9.51)$ & 115 & $14.56(7.37)$ \\
\hline Elaboración & 204 & $13.90(7.06)$ & 168 & $15.03(7.35)$ & 146 & $13.28(9.58)$ & 115 & $16(8.90)$ \\
\hline
\end{tabular}

Los alumnos de $2^{\circ}$ de ESO obtuvieron medias más elevadas en la variable de pensamiento divergente y los alumnos de $4^{\circ}$ de ESO puntuaron más alto que el resto de cursos en elaboración. Una vez realizado el análisis de varianza, los resultados 
evidenciaron diferencias estadísticamente significativas en las variables de creatividad (ver tabla 4).

Tabla 7. Diferencias entre las variables estudiadas según curso académico

\begin{tabular}{lcccc}
\hline \multicolumn{1}{c}{ Variable dependiente } & Gl & F & Sig. & Diferencias entre grupos \\
\hline Pensamiento divergente* & $3,576.962$ & 4.567 & $\mathbf{. 0 0 4}$ & $1>4,2>4$ \\
\hline Elaboración* & $3,505.602$ & 2.834 & $\mathbf{. 0 3 8}$ & $3<4$ \\
\hline *Se presenta el valor de Brown-Forsythe al no cumplirse el supuesto de homogeneidad de varianzas
\end{tabular}

Los análisis realizados a posteriori mostraron diferencias en pensamiento divergente entre los alumnos de $1^{\circ}$ de ESO y $4^{\text {a }}$ de ESO a favor de los de primero, también resultaron significativas las diferencias en creatividad entre los alumnos de $2^{\circ}$ y 4 de ESO a favor de los de segundo. En relación al factor elaboración, se halló diferencias en las puntuaciones en $3^{\circ}$ y $4^{\circ}$ de ESO a favor de los segundos que obtienen resultados más altos (ver tabla 7 y gráfica 3 ).
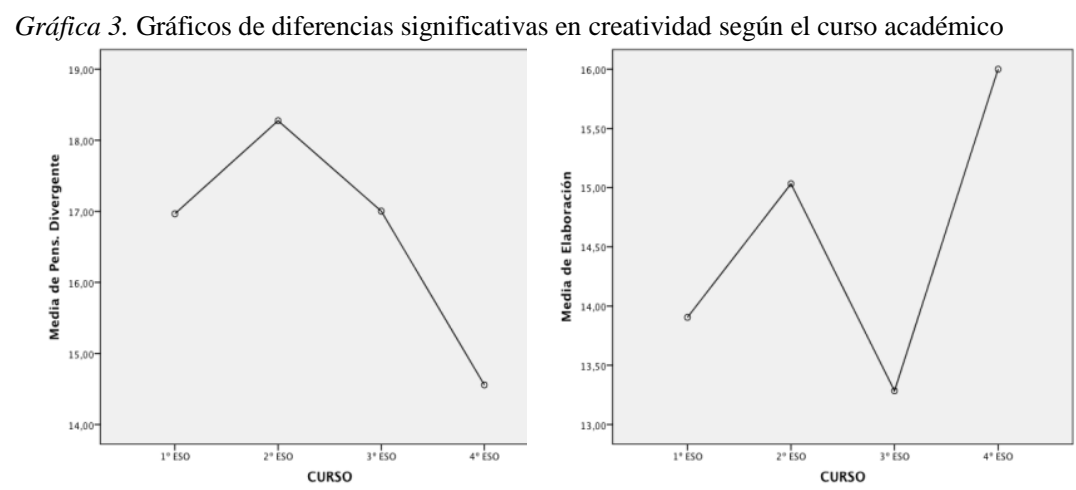

\section{DISCUSIÓN Y CONCLUSIONES}

En primer lugar, hacemos constatar que la medición del constructo de creatividad es difícil y no está exenta de controversia. En nuestra investigación, partimos de un enfoque psicométrico de la misma (Guilford, 1967; Torrance, 1974) que permite la delimitación factorial. Se utilizó por ello la subprueba de "líneas paralelas" de la parte figurativa del TCTT. Ésta se ha mostrado en diferentes investigaciones como un factor aislado que además explicaba la mayor varianza en la prueba (alrededor del 40\%) (Ferrando et al., 2007; Prieto et al., 2006). En dichas investigaciones quedaba de manifiesto que cada tarea del test figurativo estaría midiendo un tipo de creatividad distinto, de acuerdo a las demandas específicas de cada tarea. Así, la originalidad evaluada en la subprueba "acabar un dibujo" (donde se dan 10 trazos distintos), es 
diferente a la originalidad medida en "líneas paralelas" (donde el alumno se enfrenta 30 veces consecutivas con el mismo estímulo al que debe ofrecer respuestas distintas) (Almeida, Prieto, Ferrando, Oliveira, y Ferrándiz, 2008; Ferrando et al., 2007; Oliveira et al., 2009; Prieto et al., 2006). Se puede afirmar que la creatividad se expresa de forma distinta según la tarea a realizar (Perkins, 2003), por ello se escogió sólo una de las tareas para la medición del constructo de creatividad.

El análisis factorial reveló que las dimensiones de fluidez, flexibilidad y originalidad se agrupan en un primer factor, que denominamos "pensamiento divergente", sin embargo, la variable elaboración satura en un segundo factor. El primero, se relaciona con la capacidad de crear ideas nuevas, originales y variadas; mientras que el segundo referencia más a la habilidad para redefinir o mejorar una idea existente. Los resultados van en consonancia con los obtenidos en estudios previos y con aquellos autores que ven que la elaboración es la variable que menos se relaciona con la creatividad (Clapham, 1998; Ferrando et al., 2007; López, 2001). En este sentido, ya Guilford (1950) apuntó a la fluidez, flexibilidad y originalidad como las dimensiones principales de la creatividad (Holling y Kuhn, 2008).

Respecto a la prueba de inteligencia (DAT-5) no hemos estudiado las características psicométricas, porque han sido validadas en otros estudios (Hernández, 2010). Sí hemos de considerar que esta prueba nos permite una medición de la inteligencia general. En este sentido, estudios como el de Almeida et al. (2010) apoyan la convergencia de las distintas áreas cognitivas en un único factor "g". Las subpruebas de ortografía y rapidez y exactitud perceptiva han sido eliminadas en la constitución de la variable de "inteligencia psicométrica", dado que se han mostrado poco relevantes para la constitución del factor "g" (Sainz, 2010).

El objetivo principal de nuestro trabajo ha planteado la posible existencia de diferencias en la creatividad en función de las variables de género, nivel intelectual y curso de los participantes.

La variable género no presentó diferencias significativas en el pensamiento divergente (que aglutina los principales factores del modelo: fluidez, flexibilidad y originalidad), confirmando resultados de otros estudios en los que no se encontró diferencias dependiendo del sexo para la prueba TTCT (Ferrando et al., 2007; Soto, 2012). Baer y Kaufman (2008) realizaron una revisión de la literatura al respecto apuntando los mismos resultados. Por su parte, la variable elaboración arrojó diferencias estadísticamente significativas, mostrando las chicas mayor capacidad personal para desarrollar y/o perfeccionar una idea o producción alcanzando mejores niveles de complejidad y detalle.

En cuanto al nivel intelectual, no se han hallado diferencias estadísticamente significativas en la variable de pensamiento divergente. Estos resultados apoyan los estudios que no corroboran la teoría del umbral e indican la independencia de los 
constructos de inteligencia y creatividad (Kim, 2005; Preckel, Holling, y Wiese, 2006; Sainz, 2010). La variable elaboración, sí muestra diferencias significativas en función de la inteligencia a favor de los grupos de mayor capacidad cognitiva. Los datos nos indican que el nivel cognitivo facilita la capacidad de mejorar, perfeccionar o adicionar elementos nuevos a una idea. No obstante, este hecho no conlleva que mejore la capacidad para generar nuevas ideas (pensamiento divergente).

Por último, en cuanto a la creatividad y la variable de curso académico, los alumnos de $2^{\circ}$ ESO obtienen los resultados más altos en el pensamiento divergente y éste decrece conforme avanzan los cursos escolares, siendo los alumnos de $4^{\circ}$ ESO los que obtienen los resultados más bajos. Por su parte, la variable de elaboración aumenta también de $1^{\circ}$ a $2^{\circ}$ de ESO, decreciendo en el $3^{\circ}$ ESO, pero al contrario que el pensamiento divergente, muestra su cénit en $4^{\circ}$ curso. En este sentido, existen estudios sobre cómo el desarrollo evolutivo influye en la creatividad. Runco y Sakamoto (1999), indica que los distintos decrecimientos que aparecen en el pensamiento creativo están relacionados con el desarrollo de los procesos cognitivos. Smith y Carlsson (1983, 1990) manifiestan que los niños necesitan llegar a una madurez cognitiva para que la creatividad ocurra, esto pasaría alrededor de los 5-6 años de edad. Se sufriría un bajón a la edad de 7-8 años y un incremento hasta la edad de 12 donde la creatividad vuelve a descender, pero ahora de forma gradual, para posteriormente ir ascendiendo hasta alcanzar un pico de creatividad a los 16 años de edad. Claxton, Pannells y Rhoads (2005) observan diferencias según la dimensión medida, experimentando el alumnado una bajada de las puntuaciones de originalidad en el sexto grado, pero incrementando sus puntuaciones respecto de la fluidez y la flexibilidad. Kim (2011) indica una disminución del pensamiento creativo en el sexto grado. En cuanto a capacidades específicas, la fluidez aumenta hasta el tercer grado y se muestra estática entre cuarto y quinto grado, y luego disminuye continuamente; mientras que la originalidad aumenta hasta el quinto grado, disminuye en la escuela secundaria, y luego vuelve a aumentar en la edad adulta. Pese a que nuestros resultados indican una relación entre edad y la creatividad, reforzando la teoría de que el proceso de desarrollo influye en la capacidad creativa, los datos no corroboran claramente ninguno de los estudios reseñados. No obstante, en general, sí se relacionan con la línea propuesta por Kim en cuanto indica que la capacidad creativa decrece durante la ESO; sin embargo, la variable de pensamiento divergente no confirma los datos de Smith y Carlsson que apuntan a un cénit a los 16 años, que sí es mostrada en la variable elaboración. Una posible explicación del aumento de la creatividad de $1^{\circ}$ a $2^{\circ}$ ESO y su posterior descenso, es que estos sujetos presentan un cambio al pensamiento hipotético-deductivo (estadio de operaciones formales siguiendo la teoría del desarrollo cognitivo de Piaget), que le permite pensar en términos de posibilidad y no solo en términos de realidad, lo que podría potenciar las nuevas ideas y perspectivas. No obstante, las exigencias escolares que conllevan en la mayoría de 
ocasiones un uso casi exclusivo de las destrezas convergentes del pensamiento irían configurando un perfil de funcionamiento que se alejaría de lo creativo. En este sentido, la elaboración puede ser mejor acogida en las aulas, dado que no implica tanto la novedad sino el perfeccionamiento de la idea, por tanto conforme la madurez actúa en los alumnos, esta destreza se desligaría de la divergencia y se utilizará para mejorar y optimizar el trabajo escolar dentro de un pensamiento más convergente.

Como síntesis, los resultados de nuestro estudio apuntan a una estructura de dos diferenciados factores en la creatividad, el pensamiento divergente y la elaboración, mostrándose éste último sensiblemente mayor entre la población femenina de la muestra, lo que indicaría un nivel algo más elevado en las chicas al desarrollar, dar complejidad y detallar una producción o idea. Por otra parte, no se hallaron diferencias en función del nivel intelectual para el factor de pensamiento divergente y sí en el factor de elaboración a favor de los grupos de mayor capacidad intelectiva, lo que apoyaría en parte la independencia de los constructos de creatividad e inteligencia. Por último, la variable de curso nos muestra un decrecimiento del pensamiento divergente y la elaboración conforme se avanza en la ESO, salvo que este último factor presentará un cénit en el último curso de la enseñanza obligatoria.

\section{REFERENCIAS}

Almeida, L., Prieto, M.D., Ferrando, M., Oliveira, E., y Ferrándiz, C. (2008). Torrance Test of Creative Thinking: the question of its construct validity. Thinking Skills and Creativity, 3, 53-58.

Baer, J., y Kaufman, J.C. (2008). Gender differences in creativity. Journal of Creative Behavior, $42,75-105$.

Bennett, G., Seashore, H.G., y Wesman, A.G. (2000). Test de Aptitudes Diferenciales (DAT-5). Madrid: TEA Ediciones.

Clapham, M.M. (1998). Structure of Figural Forms A and B of The Torrance Tests of Creative Thinking. Educational y Psychological Measurement, 58(2), 275-283.

Claxton, A.F., Pannells, T.C., y Rhoads, P.A. (2005). Developmental Trends in the Creativity of School-Age Children. Creativity Research Journal, 17(4), 327-335.

Ferrando, M., Ferrándiz, C., Bermejo García, R., Sánchez, C., Parra, J., y Prieto, M.D. (2007). Estructura interna y baremación del Test de Pensamiento Creativo de Torrance. Psicothema, 19(3), 489-496.

Guilford, J.P. (1950). Creativity. American Psychologist, 5, 444-454.

Guilford, J.P. (1967). The Nature of Human Intelligence. New York: McGraw-Hill.

Holling, H. y Kuhn J.T. (2008). Does intellectual giftedness affect the factor structure of divergent thinking? Evidence form a MG-MACS analisis. Psychology Science Quartely, 50(2), 283-294.

Kim, K.H. (2005). Can Only Intelligence People Be Creative? The Journal of Secondary Gifted Education, $X V I(2 / 3), 57-66$.

Kim, K.H. (2011). The Creativity Crisis: The Decrease in Creative Thinking Scores on the Torrance Tests of Creative Thinking, Creativity Research Journal, 23(4), 285-295. 
López-Martínez, O. y Navarro-Lozano, J. (2010). Creatividad e inteligencia: un estudio en Educación Primaria. Revista de Investigación Educativa, 28(2), 283-296.

López, O. (2001). Evaluación y Desarrollo de la Creatividad. Tesis Doctoral. Universidad de Murcia.

Miranda, L., Almeida, L.S., Morais, F., y Guisande, M.A. (2012). Creatividad, inteligencia y rendimiento escolar: Estudio de las relaciones recíprocas en una muestra de $6^{\circ}$ año de escolaridad. Faisca, 16(18), 68-83.

Naderi, H., Abdullah, R., Aizan, H.T., Sharir, J., y Kumar, V. (2010). Creativity, Age And Gender As Predictors Of Academic Achievement Among Undergraduate Students. Journal of American Science, 5(5), 101-112.

Nusbaum, E.C. y Silvia, P.J. (2010). Are intelligence and creativity really so different? Fluid intelligence, executive processes, and strategy use in divergent thinking. Intelligence, 39 , 36-45.

Oliveira, E.P., Almeida, L., Ferrándiz, C., Ferrando, M., Sáinz, M., y Prieto, M.D. (2009). Tests de Pensamiento Creativo de Torrance (TTCT): Elementos para la validez del constructo en adolescentes portugueses. Psicothema, 21(4), 562-567.

Perkins (2003). La bañera de Arquímedes y otras historias del pensamiento creativo. El arte del pensar creativo. Barcelona: Paidós.

Preckel, F. Holling, H., y Wiese, M. (2006). Intelligence and creativity in gifted and non-gifted students: An investigation of threshold theory. Personality and Individual Differences, 40, 159-170.

Prieto, M.D., Parra, J., Ferrando, M., Ferrándiz, C., Bermejo, M.R., y Sánchez, C. (2006). Creative habilities in early childhood. Journal of Early Childhood Research, 4(3), 227-290.

Runco, M. y Sakamoto, S. (1999). Experimentals studies of creativity. En R.J. Sternberg (Ed.), Handbook of Creativity. New York: Cambridge University Press.

Sáinz, M. (2010). Creatividad, personalidad y competencia socio emocional en alumnos de altas habilidades versus no altas habilidades. Tesis Doctoral. Universidad de Murcia.

Silvia, P.J. (2008). Creativity and intelligence revisited: A reanalysis of Wallach and Kogan (1965). Creativity Research Journal, 20, 34-39.

Silvia, P.J. y Roger, E.B. (2012) Making creative metaphors: The importance of fluid intelligence for creative thought. Intelligence, 40(4), 343-351.

Smith, G.J., y Carlsson, I.M. (1983). Creativity in early and middle school years. International Journal of Behavioral Development, 6, 167-195.

Smith, G.J., y Carlsson, I.M. (1990). The creative process: Afunctional model based on empirical studies from early childhood to middle age. Madison, CT: International Universities Press.

Soto, G. (2012). Diferentes perspectivas para evaluar el pensamiento creativo. Tesis Doctoral. Universidad de Murcia.

Torrance, E.P. (1974). The Torrance Tests of Creative Thinking - Norms-Technical Manual Research Edition - Verbal Tests, Forms A and B - Figural Tests, Forms A and B. Princeton NJ: Personnel Press.

Yuste, C., Martínez, R., y Galvez, J.L. (1998). Batería de aptitudes diferenciales y generales (BADyG-E3). Madrid: CEPE.

Recibido: 2 de septiembre de 2017 Recepción Modificaciones: 23 de octubre de 2017

Aceptado: 29 de octubre de 2017 